\title{
Some qualitative properties of linear dynamic equations with multiple delays
}

\section{Jan Čermák}

${ }^{\text {"Correspondence: }}$

cermak.j@fme.vutbr.cz Institute of Mathematics, Brno

University of Technology,

Technická 2, Brno, 616 69, Czech

Republic

\begin{abstract}
This paper discusses stability and asymptotic properties of the delay dynamic equation

$$
y^{\nabla}(t)=\sum_{j=0}^{k} a_{j} y\left(\tau^{j}(t)\right), \quad t \in \mathbb{T},
$$

where $a_{j} \in \mathbb{R}$ are scalars, $\tau^{j}$ are iterates of a function $\tau: \mathbb{T} \rightarrow \mathbb{T}$ and $\mathbb{T}$ is a time scale unbounded above. Under some specific choices of $\tau$, this dynamic equation involves several significant particular cases such as linear autonomous differential equations with several delays or linear autonomous higher-order difference equations. For proportional $\tau$, we formulate an asymptotic result joint for two different time scales, including a joint form of its proof. For a general $\tau$, we investigate stability and asymptotic properties of solutions on the continuous and discrete time scales separately. Besides a related character of the relevant results, we discuss also a possible related character of their proofs.
\end{abstract}

MSC: 34N05; 34K25; 39A12; 39A30

Keywords: delay dynamic equation; stability; asymptotic behaviour

\section{Introduction}

We consider the delay dynamic equation

$$
y^{\nabla}(t)=\sum_{j=0}^{k} a_{j} y\left(\tau^{j}(t)\right), \quad t \in \mathbb{T},
$$

where $a_{j}$ are real scalars, $\tau^{j}$ are the $j$ th iterates of an increasing function $\tau: \mathbb{T} \rightarrow \mathbb{T}$ with $\tau^{0} \equiv \mathrm{id}, \tau^{1} \equiv \tau, \tau(t)<t$ for $t \in \mathbb{T}$ and $\mathbb{T}$ is a time scale unbounded above. If $\mathbb{T}$ has a finite minimum $m$, we admit $\tau(m)=m$.

Qualitative analysis of delay dynamic equations on time scales has already been the subject of several investigations (see, e.g. [1-4] and [5]). In particular, these papers present techniques which enable a joint analysis of delay differential and difference equations. Besides the methods developed directly for delay dynamic equations, there are also some proof procedures utilised originally either for delay differential or difference equations, but they seem to be applicable without any extra difficulties also to a general dynamic case (see, e.g. [6] and [7]).

\section{照 Springer}

๑ 2013 Čermák; licensee Springer. This is an Open Access article distributed under the terms of the Creative Commons Attribution License (http://creativecommons.org/licenses/by/2.0), which permits unrestricted use, distribution, and reproduction in any medium, provided the original work is properly cited. 
In this paper, we discuss common stability and asymptotic properties of the delay dynamic equation (1.1). Although (1.1) can be taken for a basic type of delay dynamic equations, its qualitative analysis has not been described yet. It might be surprising because delay dynamic equations studied in the above mentioned papers mostly have more complicated forms. A difficulty connected with qualitative analysis of (1.1) follows from the proof methods utilised in its significant particular cases, which are of a quite different nature, and their unification to a general dynamic case can be very complicated. Nevertheless, considering (1.1) with unbounded lags, we describe the cases when a joint investigation of (1.1) is possible.

This paper is organised as follows. In Section 2 we introduce several crucial particular cases of (1.1). Section 3 is devoted to asymptotic estimation of the dynamic pantograph equation. These estimates are generalised and improved in Section 4. Some final remarks conclude the paper.

\section{Preliminaries}

Throughout this paper we adopt the standard time scale notation. In particular, $\rho: \mathbb{T} \rightarrow \mathbb{T}$ is the backward jump operator, $v: \mathbb{T} \rightarrow \mathbb{R}_{0}^{+}$is the backward graininess and the symbol $y^{\nabla}$ means the nabla derivative of $y: \mathbb{T} \rightarrow \mathbb{R}$. For precise introductions of these symbols and other related matters, we refer to [8] and [9].

By a solution of (1.1), we mean a function $y: \mathbb{T} \rightarrow \mathbb{R}$ which is ld-continuous on $\left[\tau^{k}\left(t_{0}\right), \infty\right)$, has an ld-continuous nabla derivative on $\left[t_{0}, \infty\right)$ and satisfies $(1.1)$ on $\left[t_{0}, \infty\right)$ for some $t_{0} \in \mathbb{T}$. In this definition (as well as throughout the whole paper), we use the classical interval notation without a specification of $\mathbb{T}(e . g .[a, b)$ means $\{t \in \mathbb{T}: a \leq t<b\})$.

We prefer to study (1.1) as a nabla dynamic equation (instead of its delta analogue) because, as stated above, our aim is to describe qualitative properties of (1.1) common to different time scales. It is well known from numerical analysis of differential equations that there are just discretisations based on backward (nabla) differences which enable to retain the key properties of underlying differential equations.

The assumption

$$
\tau: \mathbb{T} \rightarrow \mathbb{T}
$$

(implying $\tau^{j}: \mathbb{T} \rightarrow \mathbb{T}$ ) is standardly employed in the papers on delay dynamic equations (for some ideas about its possible removing, we refer to [10]). While for $\mathbb{T}=\mathbb{R}$ this assumption becomes trivial, for discrete time scales (i.e. time scales with a nonzero graininess) it represents a considerable restriction. More precisely, a concrete choice of $\tau$ significantly influences the form of $\mathbb{T}$ (and vice versa). Therefore we distinguish the following cases.

(a) Equation (1.1) with constant lags.

If $\tau(t)=t-h, h>0$, then besides $\mathbb{T}=\mathbb{R}$ the only 'natural' discrete time scale preserving (2.1) is the set $\mathbb{T}=h \mathbb{Z}=\{n h: n \in \mathbb{Z}\}$. Using a scaling of the independent variable, we can fix the lag $h$ equal to 1 ; hence without loosing a generality, we put $h=1$. Then, for $\mathbb{T}=\mathbb{R}$, (1.1) becomes the linear autonomous differential equation with multiple delays

$$
y^{\prime}(t)=\sum_{j=0}^{k} a_{j} y(t-j), \quad t \in \mathbb{R},
$$


while for $\mathbb{T}=\mathbb{Z}$, (1.1) turns into

$$
y(t)-y(t-1)=\sum_{j=0}^{k} a_{j} y(t-j), \quad t \in \mathbb{Z},
$$

which is a linear autonomous higher-order difference equation (both terms on its lefthand side can be obviously joined to the right-hand side to obtain the standard general form of such an equation, see, e.g. [11]).

A key property discussed in connection with (2.2) and (2.3) is their asymptotic stability; more precisely, a formulation of necessary and sufficient conditions guaranteeing that any solution $y$ is tending to zero as $t \rightarrow \infty$. The standard way to investigate this property originates from the zero analysis of characteristic polynomials. If $\mathbb{T}=\mathbb{R}$ then substituting $y(t)=\exp (\lambda t)$ into (2.2), we are led to the quasipolynomial

$$
P(\lambda) \equiv \lambda-\sum_{j=0}^{k} a_{j} \exp (-j \lambda) .
$$

If $\mathbb{T}=\mathbb{Z}$ then putting $y(t)=\lambda^{t}$ in (2.3), we obtain the classical polynomial

$$
Q(\lambda) \equiv \sum_{j=0}^{k} a_{j} \lambda^{k-j}
$$

(where we replaced $a_{0}-1$ by $a_{0}$ and $a_{1}+1$ by $a_{1}$ ). In the first case, we need to formulate necessary and sufficient conditions ensuring that all zeros of $P(\lambda)$ lie in the left half of the complex plane. In the second case, we seek necessary and sufficient conditions for all zeros of $Q(\lambda)$ to be located inside the unique circle. The problem concerning zeros of the quasipolynomial $P(\lambda)$ is original and must be solved by specific procedures (see, e.g. [12]). The matter concerning zeros of $Q(\lambda)$ is a classical polynomial problem. In a theoretical level, it can be solved by use of the Schur-Cohn test or the Jury criterion (see [13]). However, a formulation of explicit necessary and sufficient conditions (in terms of $a_{j}$ and $k$ ) is still an open problem. From this viewpoint, a unification of both procedures and formulation of explicit necessary and sufficient conditions for the asymptotic stability of the delay dynamic equation

$$
y^{\nabla}(t)=\sum_{j=0}^{k} a_{j} y(t-j), \quad t \in \mathbb{T},
$$

generalising (2.2) and (2.3), seem to be an extremely difficult matter. Therefore, we are going to pay our attention to other types of delays.

(b) Equation (1.1) with proportional lags.

If $\tau(t)=q^{-1} t, q>1$, then besides $\mathbb{T}=\mathbb{R}_{0}^{+}$we consider the discrete time scale $\mathbb{T}=\overline{q^{\mathbb{Z}}}=$ $\left\{q^{n}: n \in \mathbb{Z}\right\} \cup\{0\}$ to satisfy the condition (2.1). For these two settings, (1.1) becomes the multipantograph equation

$$
y^{\prime}(t)=\sum_{j=0}^{k} a_{j} y\left(q^{-j} t\right), \quad t \in \mathbb{R}_{0}^{+}
$$


and its $q$-analogue

$$
\nabla_{q} y(t)=\sum_{j=0}^{k} a_{j} y\left(q^{-j} t\right), \quad t \in \overline{q^{\mathbb{Z}}},
$$

where

$$
\nabla_{q} y(t)=\frac{y(t)-y\left(q^{-1} t\right)}{\left(1-q^{-1}\right) t}
$$

is the backward Jackson derivative. There are different techniques how to analyse stability and asymptotic properties of differential equations of the pantograph type. The most frequent one is the method of Dirichlet series (see, e.g. [14] and [15]), which is, however, not applicable to the corresponding dynamic equation

$$
y^{\nabla}(t)=\sum_{j=0}^{k} a_{j} y\left(q^{-j} t\right), \quad t \in \mathbb{T} .
$$

Fortunately, among other techniques on pantograph differential equations, there exists a method which can be extended also to (2.7). It originates from the pioneering paper [16] on differential equations of this type, and in the next section we introduce its extended modified version to prove an asymptotic result for (2.7).

(c) Equation (1.1) with general lags.

If the form of $\tau$ is not specified, then (2.1) does not imply any concrete restriction. Nevertheless, previous special dynamic equations (2.4) and (2.7) can inspire us to meet the condition (2.1) 'implicitly' by considering the dynamic equation

$$
y^{\nabla}(t)=\sum_{j=0}^{k} a_{j} y\left(\rho^{j}(t)\right), \quad t \in \mathbb{T},
$$

instead of (1.1). Indeed, if $\mathbb{T}=\mathbb{Z}$ then (2.8) becomes (2.3), and for $\mathbb{T}=\overline{q^{\mathbb{Z}}}$ (2.8) becomes (2.6). In other words, the role of delayed arguments in (2.8) is played by the backward jump operator and its iterates. In Section 4, we give a precise asymptotic description for the solutions of (2.8), including an asymptotic stability condition.

\section{A dynamic equation with proportional delays}

Let $a_{0}<0$ and $a_{k} \neq 0$. We consider the polynomial

$$
\tilde{Q}(\lambda) \equiv a_{0} \lambda^{k}+\sum_{j=1}^{k}\left|a_{j}\right| \lambda^{k-j}
$$

and make some simple observations on its real zeros. Since $\tilde{Q}(0)=\left|a_{k}\right|>0$ and $\tilde{Q}(\lambda) \rightarrow$ $-\infty$ as $\lambda \rightarrow \infty, \tilde{Q}(\lambda)$ has a positive real zero. We show its uniqueness. Let $\lambda_{*}$ be a positive zero of $\tilde{Q}(\lambda)$, i.e. $\tilde{Q}\left(\lambda_{*}\right)=0$. Then it is easy to check that $\tilde{Q}^{\prime}\left(\lambda_{*}\right)<0$, which along with $\tilde{Q}(0)>0$ implies that such a positive zero must be unique. We denote it by $\lambda_{r}$ and add that 
$0<\lambda_{r}<1$ if and only if

$$
\sum_{j=1}^{k}\left|a_{j}\right|<-a_{0}
$$

Using this notation, we have the following.

Theorem 3.1 Let $y$ be a solution of (2.7), where $a_{0}<0, a_{k} \neq 0, q>1$ and let $\mathbb{T}=\mathbb{R}_{0}^{+}$or $\mathbb{T}=\overline{q^{\mathbb{Z}}}$. Then

$$
y(t)=O\left(t^{r}\right) \quad \text { as } t \rightarrow \infty, \quad r=\log _{q} \lambda_{r} .
$$

Proof All necessary formulae of the basic time scales calculus utilised below (such as product rule, quotient rule or integration by parts) can be found in [8], pp.331-333. Similarly, we use the symbol $\hat{\mathrm{e}}_{a_{0}}\left(t, t_{0}\right)$ as the nabla exponential function satisfying

$$
\hat{\mathrm{e}}_{a_{0}}^{\nabla}\left(t, t_{0}\right)=a_{0} \hat{\mathrm{e}}_{a_{0}}\left(t, t_{0}\right), \quad \hat{\mathrm{e}}_{a_{0}}\left(t_{0}, t_{0}\right)=1
$$

(for its precise introduction and properties, we refer to [9], pp.49-55). In particular, the assumption $a_{0}<0$ implies $1-a_{0} v(t)>0$ for all $t \in \mathbb{T}$ (positive regressivity), i.e. $\hat{\mathrm{e}}_{a_{0}}\left(t, t_{0}\right)$ is positive on $\mathbb{T}$.

Let $y$ satisfy $(2.7)$ on $\left[t_{0}, \infty\right)$ for some $t_{0} \in \mathbb{T}, t_{0}>0$. Put $z(t)=y(t) / t^{r}, t \in \mathbb{T}, t \geq q^{-k} t_{0}$. Substituting into (2.7), we have

$$
z^{\nabla}(t) t^{r}+z(\rho(t))\left(t^{r}\right)^{\nabla}=\sum_{j=0}^{k} a_{j} z\left(q^{-j} t\right)\left(q^{-j} t\right)^{r}
$$

Dividing by the integrating factor $\hat{\mathrm{e}}_{a_{0}}\left(\rho(t), t_{0}\right)$, one gets

$$
\left[\frac{z(t) t^{r}}{\hat{\mathrm{e}}_{a_{0}}\left(t, t_{0}\right)}\right]^{\nabla}=\frac{\sum_{j=1}^{k} a_{j} z\left(q^{-j} t\right)\left(q^{-j} t\right)^{r}}{\hat{\mathrm{e}}_{a_{0}}\left(\rho(t), t_{0}\right)}
$$

Now we put $t_{-1}=q^{-k} t_{0}, t_{i}=q^{i} t_{0}$ and $S_{i}:=\sup \left\{|z(t)|: t \in\left[t_{-1}, t_{i}\right]\right\}, i=1,2, \ldots$ Let $u \in$ $\left[t_{i}, t_{i+1}\right]$ be arbitrary. Integrating the previous relation over $\left[t_{i}, u\right]$, we obtain

$$
\frac{z(u) u^{r}}{\hat{\mathrm{e}}_{a_{0}}\left(u, t_{0}\right)}-\frac{z\left(t_{i}\right)\left(t_{i}\right)^{r}}{\hat{\mathrm{e}}_{a_{0}}\left(t_{i}, t_{0}\right)}=\int_{t_{i}}^{u} \frac{\sum_{j=1}^{k} a_{j} z\left(q^{-j} t\right)\left(q^{-j} t\right)^{r}}{\hat{\mathrm{e}}_{a_{0}}\left(\rho(t), t_{0}\right)} \nabla t,
$$

which implies

$$
z(u)=\frac{\left(t_{i}\right)^{r} \hat{\mathrm{e}}_{a_{0}}\left(u, t_{0}\right)}{u^{r} \hat{\mathrm{e}}_{a_{0}}\left(t_{i}, t_{0}\right)} z\left(t_{i}\right)+\frac{\hat{\mathrm{e}}_{a_{0}}\left(u, t_{0}\right)}{u^{r}} \int_{t_{i}}^{u} \frac{\sum_{j=1}^{k} a_{j} z\left(q^{-j} t\right)\left(q^{-j} t\right)^{r}}{\hat{\mathrm{e}}_{a_{0}}\left(\rho(t), t_{0}\right)} \nabla t,
$$

i.e.

$$
|z(u)| \leq S_{i} \frac{\left(t_{i}\right)^{r} \hat{\mathrm{e}}_{a_{0}}\left(u, t_{0}\right)}{u^{r} \hat{\mathrm{e}}_{a_{0}}\left(t_{i}, t_{0}\right)}+S_{i} \frac{\hat{\mathrm{e}}_{a_{0}}\left(u, t_{0}\right)}{u^{r}} \int_{t_{i}}^{u} \frac{\sum_{j=1}^{k}\left|a_{j}\right|\left(q^{-j} t\right)^{r}}{\hat{\mathrm{e}}_{a_{0}}\left(\rho(t), t_{0}\right)} \nabla t .
$$


Since

$$
a_{0} t^{r}+\sum_{j=1}^{k}\left|a_{j}\right|\left(q^{-j} t\right)^{r}=0
$$

we can write

$$
|z(u)| \leq S_{i} \frac{\left(t_{i}\right)^{r} \hat{\mathrm{e}}_{a_{0}}\left(u, t_{0}\right)}{u^{r} \hat{\mathrm{e}}_{a_{0}}\left(t_{i}, t_{0}\right)}+S_{i} \frac{\hat{\mathrm{e}}_{a_{0}}\left(u, t_{0}\right)}{u^{r}} \int_{t_{i}}^{u} \frac{-a_{0} t^{r}}{\hat{\mathrm{e}}_{a_{0}}\left(\rho(t), t_{0}\right)} \nabla t
$$

i.e.

$$
|z(u)| \leq S_{i} \frac{\left(t_{i}\right)^{r} \hat{\mathrm{e}}_{a_{0}}\left(u, t_{0}\right)}{u^{r} \hat{\mathrm{e}}_{a_{0}}\left(t_{i}, t_{0}\right)}+S_{i} \frac{\hat{\mathrm{e}}_{a_{0}}\left(u, t_{0}\right)}{u^{r}} \int_{t_{i}}^{u}\left[\frac{1}{\hat{\mathrm{e}}_{a_{0}}\left(t, t_{0}\right)}\right]^{\nabla} t^{r} \nabla t .
$$

The integration by parts yields

$$
\int_{t_{i}}^{u}\left[\frac{1}{\hat{\mathrm{e}}_{a_{0}}\left(t, t_{0}\right)}\right]^{\nabla} t^{r} \nabla t=\left[\frac{t^{r}}{\hat{\mathrm{e}}_{a_{0}}\left(t, t_{0}\right)}\right]_{t_{i}}^{u}-\int_{t_{i}}^{u}\left(t^{r}\right)^{\nabla} \frac{1}{\hat{\mathrm{e}}_{a_{0}}\left(\rho(t), t_{0}\right)} \nabla t
$$

Now let (3.1) hold. Then $r<0$ and $\left(t^{r}\right)^{\nabla}$ is increasing for both the time scales $\mathbb{T}=\mathbb{R}_{0}^{+}$and $\mathbb{T}=\overline{q^{\mathbb{Z}}}$, hence

$$
\int_{t_{i}}^{u}\left(t^{r}\right)^{\nabla} \frac{1}{\hat{\mathrm{e}}_{a_{0}}\left(\rho(t), t_{0}\right)} \nabla t \geq \frac{\left.\left(t^{r}\right)^{\nabla}\right|_{t=t_{i}}}{-a_{0}} \int_{t_{i}}^{u} \frac{-a_{0}}{\hat{\mathrm{e}}_{a_{0}}\left(\rho(t), t_{0}\right)} \nabla t=\frac{\left.\left(t^{r}\right)^{\nabla}\right|_{t=t_{i}}}{-a_{0}}\left[\frac{1}{\hat{\mathrm{e}}_{a_{0}}\left(t, t_{0}\right)}\right]_{t_{i}}^{u} .
$$

We involve this estimate into (3.4) and obtain

$$
|z(u)| \leq S_{i}+S_{i} \frac{\hat{\mathrm{e}}_{a_{0}}\left(u, t_{0}\right)}{u^{r}} \frac{\left.\left(t^{r}\right)^{\nabla}\right|_{t=t_{i}}}{-a_{0}}\left[\frac{1}{\hat{\mathrm{e}}_{a_{0}}\left(t, t_{0}\right)}\right]_{t_{i}}^{u} \leq S_{i}\left(1-\frac{\left.\left(t^{r}\right)^{\nabla}\right|_{t=t_{i}}}{-a_{0}\left(t_{i+1}\right)^{r}}\right) .
$$

Since $u \in\left[t_{i}, t_{i+1}\right]$ was arbitrary,

$$
S_{i+1} \leq S_{i}\left(1-\frac{\left.\left(t^{r}\right)^{\nabla}\right|_{t=t_{i}}}{-a_{0}\left(t_{i+1}\right)^{r}}\right) \leq S_{1} \prod_{\ell=1}^{i}\left(1-\frac{\left.\left(t^{r}\right)^{\nabla}\right|_{t=t_{\ell}}}{-a_{0}\left(t_{\ell+1}\right)^{r}}\right) .
$$

A straightforward calculation yields that

$$
\frac{\left.\left(t^{r}\right)^{\nabla}\right|_{t=t_{\ell}}}{a_{0}\left(t_{\ell+1}\right)^{r}}=O\left(q^{-\ell}\right) \quad \text { as } \ell \rightarrow \infty
$$

for both the time scales $\mathbb{T}=\mathbb{R}_{0}^{+}$and $\mathbb{T}=\overline{q^{\mathbb{Z}}}$, hence the product in (3.6) converges as $i \rightarrow \infty$, i.e. $z$ is bounded. This proves the asymptotic property (3.2).

The case when (3.1) does not hold, i.e. the case $r \geq 0$, is a simplified version of the previous one. Since $\left(t^{r}\right)^{\nabla}$ is nonnegative in such a case, we can deduce from (3.5) that

$$
\int_{t_{m}}^{u}\left[\frac{1}{\hat{\mathrm{e}}_{a_{0}}\left(t, t_{0}\right)}\right]^{\nabla} t^{r} \nabla t \leq\left[\frac{t^{r}}{\hat{\mathrm{e}}_{a_{0}}\left(t, t_{0}\right)}\right]_{t_{i}}^{u},
$$

and using the same way as above, we arrive at $S_{i+1} \leq S_{i}$. 
Remark 3.2 The assumption $\mathbb{T}=\mathbb{R}_{0}^{+}$or $\mathbb{T}=\overline{q^{\mathbb{Z}}}$ was employed within the previous proof only in a verification $\left(t^{r}\right)^{\nabla}$ is increasing when $r<0$, and in a check on the asymptotic estimate (3.7). All other utilised proof procedures were independent of a given time scale. To the author's knowledge, the presented result is new for both the time scales (particularly, for the differential equation (2.5) when $\mathbb{T}=\mathbb{R}_{0}^{+}$).

\section{A dynamic equation with general delays}

In this section, we discuss the problem when a type of lags is not specified, i.e. we consider the delay dynamic equation (1.1). Our aim is to extend the asymptotic result formulated in Theorem 3.1.

First let $\mathbb{T}=\mathbb{R}$, i.e. we consider the delay differential equation

$$
y^{\prime}(t)=\sum_{j=0}^{k} a_{j} y\left(\tau^{j}(t)\right), \quad t \in I,
$$

where $I$ is a real interval unbounded above. A very useful method converting differential equations with general lags into equations with prescribed lags is based on the utilisation of suitable functional equations (see [17] and [18]). If the prescribed lags are constant, then the corresponding functional equation is that of Abel (for an elegant application of this approach in the oscillation theory of delay differential equations, we refer, e.g. to [19]). In our case, the prescribed lags are proportional, hence along with (4.1), we consider also the linearisation equation

$$
\varphi(\tau(t))=q^{-1} \varphi(t), \quad t \in I,
$$

which is called the Schröder equation. Let $I=[m, \infty), \tau \in C^{1}(I), \tau(m)=m, \tau(t)<t$ for all $t>m, \tau^{\prime}$ is positive and nonincreasing on $I$ and $q=1 / \tau^{\prime}(m)>1$. Then there exists a solution $\varphi \in C^{1}(I)$ of $(4.2)$, which is positive on $(m, \infty)$ and has a positive and nonincreasing derivative on $I$. For this and other relevant results on the Schröder equation, we refer to [20].

In the next assertion, we assume that $\tau$ and $\varphi$ have the properties stated above and $\lambda_{r}$ has the same meaning as in Theorem 3.1 (see the discussion preceding this theorem).

Theorem 4.1 Let $y$ be a solution of (4.1), where $a_{0}<0$ and $a_{k} \neq 0$. Then

$$
y(t)=O\left((\varphi(t))^{r}\right) \quad \text { as } t \rightarrow \infty, \quad r=\log _{q} \lambda_{r} .
$$

Proof We give only its brief outline. It is enough to replace the function $t^{r}$ by $(\varphi(t))^{r}$ and follow the proof of Theorem 3.1. Indeed, if we put $z(t)=y(t) /(\varphi(t))^{r}, t_{-1}=\tau^{k}\left(t_{0}\right)$ and $t_{i}=$ $\tau^{-i}\left(t_{0}\right)$, where $i=1,2, \ldots$, then the proof procedures presented above can be repeated step by step. In particular, (3.3) is replaced by

$$
a_{0}(\varphi(t))^{r}+\sum_{j=1}^{k}\left|a_{j}\right|\left(\varphi\left(\tau^{j}(t)\right)\right)^{r}=0
$$


which is satisfied just due to (4.2). Thus we arrive at

$$
S_{i+1} \leq S_{1} \prod_{\ell=1}^{i}\left(1-\frac{\left.\left((\varphi(t))^{r}\right)^{\prime}\right|_{t=t_{\ell}}}{-a_{0}\left(\varphi\left(t_{\ell+1}\right)\right)^{r}}\right) \leq S_{1} \prod_{\ell=1}^{i}\left(1-\frac{r}{-a_{0} q^{r}} \frac{\varphi^{\prime}\left(t_{\ell}\right)}{\varphi\left(t_{\ell}\right)}\right)
$$

instead of (3.6). Since $\varphi^{\prime}$ is bounded,

$$
\frac{\varphi^{\prime}\left(t_{\ell}\right)}{\varphi\left(t_{\ell}\right)}=O\left(q^{-\ell}\right) \quad \text { as } \ell \rightarrow \infty
$$

The remaining parts of the proof are identical with those stated above.

Remark 4.2 If $\tau(t)=q^{-1} t$, then (4.2) is satisfied by the identity function. Consequently, Theorem 4.1 is a direct generalisation of Theorem 3.1 (when $\mathbb{T}=\mathbb{R}_{0}^{+}$). Moreover, the above stated assumptions on $\tau$ particularly imply that

$$
\tau^{\prime}(t) \leq q^{-1}<1 \quad \text { for all } t \in I
$$

i.e. the lags $t-\tau^{j}(t), j=1,2, \ldots, k$ are unbounded as $t \rightarrow \infty$.

A reformulation of Theorem 4.1 and its proof for discrete time scales is not straightforward (e.g. we have used here the chain rule which is not valid on a general time scale). Therefore, we do not follow this way and give a simple alternative proof of a related assertion for discrete time scales. This assertion does not only extend, but even improves the asymptotic property (4.3).

Considering discrete time scales only, we investigate the delay dynamic equation (2.8) instead of (1.1). Therefore, instead of (4.2) we consider the Schröder equation

$$
\varphi(\rho(t))=q^{-1} \varphi(t), \quad t \in \mathbb{T} .
$$

Also, as a direct analogue to (4.4), we assume that

$$
\rho^{\nabla}(t) \leq q^{-1}<1 \quad \text { for all } t \in \mathbb{T} \text {. }
$$

Since $\rho$ is increasing, (4.5) has a positive and increasing solution $\varphi$.

The reason why the asymptotic formula (4.3) (as well as (3.2)) does not seem to be quite optimal, consists in the fact that (4.3) involves the zero analysis of the polynomial $\tilde{Q}(\lambda)$. However, the polynomial $Q(\lambda)$, whose coefficients are in a direct correspondence with coefficients of studied equations, may be more natural than $\tilde{Q}(\lambda)$. Therefore, our next aim is to clarify this correspondence. Before doing this, we state some preliminaries on zeros of $Q(\lambda)$ and their relationship to the corresponding difference equation

$$
\sum_{j=0}^{k} a_{j} \omega(s-j)=0
$$

For any positive real $\theta$, we introduce the set $\Lambda(\theta)$ of all complex zeros of $Q(\lambda)$ with the modulus $\theta$. If $\Lambda(\theta)$ is nonempty for a given $\theta$, then by a characteristic solution of (4.7) 
corresponding to $\Lambda(\theta)$, we understand a finite sum of solutions of (4.7) corresponding to all values $\lambda \in \Lambda(\theta)$. Of course, the form of such solutions depends on multiplicity of $\lambda$ and it is described in details e.g. in [11].

Using this we have the following theorem.

Theorem 4.3 Let $y$ be a solution of (2.8), where $a_{0}, a_{k} \neq 0$ and let $\mathbb{T}$ be a discrete time scale such that $a_{0} v(t) \neq 1$ for all $t \in \mathbb{T}$ and (4.6) holds for some $q>1$. Then there exists $\theta>0$ such that $\Lambda(\theta)$ is nonempty and

$$
y(t)=\omega\left(\log _{q} \varphi(t)\right)+O\left((\varphi(t))^{\gamma}\right) \quad \text { as } t \rightarrow \infty, \quad \gamma=\log _{q}(\theta-\varepsilon)
$$

where $\omega$ is a nontrivial characteristic solution of (4.7) corresponding to $\Lambda(\theta), \varphi$ is a positive and increasing solution of (4.5) and $0<\varepsilon<\theta$ is a suitable real scalar.

Proof Let $y$ satisfy $(2.8)$ on $\left[t_{0}, \infty\right)$ for some $t_{0} \in \mathbb{T}$. By the definition,

$$
\frac{y(t)-y(\rho(t))}{v(t)}=\sum_{j=0}^{k} a_{j} y\left(\rho^{j}(t)\right), \quad t \in \mathbb{T}, \quad t \geq t_{0} .
$$

Since

$$
\rho^{\nabla}(t)=\frac{\rho(t)-\rho^{2}(t)}{t-\rho(t)}=\frac{v(\rho(t))}{v(t)}
$$

it holds $v(\rho(t)) \leq q^{-1} v(t)$ in view of (4.6). Comparing with (4.5), one gets

$$
\varphi(t)=O(v(t)) \quad \text { as } t \rightarrow \infty
$$

Now put $s=\log _{q} \varphi(t)$ and $z(s)=y(t), t \in \mathbb{T}, t \geq t_{0}$. Then $y(\rho(t))=z(s-1)$ and, more generally, $y\left(\rho^{j}(t)\right)=z(s-j)$ by use of (4.5). Thus (4.9) becomes

$$
\frac{z(s)-z(s-1)}{v\left(\varphi^{-1}\left(q^{s}\right)\right)}=\sum_{j=0}^{k} a_{j} z(s-j), \quad s \in \mathbb{Z}, s \geq s_{0},
$$

$s_{0}=\log _{q} \varphi\left(t_{0}\right)$. Rewrite it as

$$
\left[a_{0}-b(s)\right] z(s)+\left[a_{1}+b(s)\right] z(s-1)+\sum_{j=2}^{k} a_{j} z(s-j)=0,
$$

where

$$
b(s)=\frac{1}{v\left(\varphi^{-1}\left(q^{s}\right)\right)}=O\left(q^{-s}\right) \quad \text { as } s \rightarrow \infty
$$

due to (4.10). Equivalently,

$$
a_{0} z(s)+\sum_{j=1}^{k}\left[a_{j}+c_{j}(s)\right] z(s-j)=0
$$


where $c_{j}(s)$ are appropriate functions satisfying $c_{j}(s)=O\left(q^{-s}\right)$ as $s \rightarrow \infty, j=1,2, \ldots, k$. Consequently, (4.11) is the higher-order difference equation of Poincaré type. Its limiting equation is just (4.7) with the characteristic polynomial $Q(\lambda)$. Moreover, the limits $a_{j}$ are approached at an exponential rate. Then, by Theorem 2.3 of [21], there exists $\theta>0$ such that $\Lambda(\theta)$ is nonempty, and for some $0<\varepsilon<\theta$, it holds

$$
z(s)=\omega(s)+O\left((\theta-\varepsilon)^{s}\right) \quad \text { as } s \rightarrow \infty,
$$

where $\omega$ is a nontrivial characteristic solution of (4.7) corresponding to $\Lambda(\theta)$. Now, using the backward substitution, we obtain (4.8).

Let $\kappa$ be a zero of $Q(\lambda)$ and $m_{\kappa}$ be its multiplicity. We call this zero maximal if $\kappa$ is (among all other zeros of $Q(\lambda)$ ) maximal in the modulus and if multiplicities of other possible zeros of $Q(\lambda)$ having the maximal modulus do not exceed $m_{\kappa}$. Of course, $Q(\lambda)$ may have several maximal zeros (with the same modulus and multiplicity).

Corollary 4.4 Let $y$ be a solution of (2.8), where $a_{0}, a_{k} \neq 0$ and let $\mathbb{T}$ be a discrete time scale such that $a_{0} v(t) \neq 1$ for all $t \in \mathbb{T}$ and (4.6) holds for some $q>1$. Further, let $\kappa$ be a maximal zero of $Q(\lambda)$ and $m_{\kappa}$ be its multiplicity. Then

$$
y(t)=O\left((\varphi(t))^{\alpha}\left(\log _{q} \varphi(t)\right)^{m_{\kappa}-1}\right) \quad \text { as } t \rightarrow \infty, \alpha=\log _{q}|\kappa|,
$$

where $\varphi$ is a positive and increasing solution of (4.5).

Remark 4.5 As an immediate consequence, we get that under the assumptions of Theorem $4.3,(2.8)$ is asymptotically stable (i.e. its any solution $y$ tends to zero as $t \rightarrow \infty$ ) if all the zeros of $Q(\lambda)$ are located inside the unite circle. In this connection, we have already mentioned the Schur-Cohn criterion, which can be applied to any polynomial $Q(\lambda)$ with concrete (fixed) coefficients and order. However, this criterion does not enable us to formulate explicit stability conditions in terms of (general) coefficients $a_{j}$ and $k$. Such explicit conditions are known only in a very few particular cases (see, e.g. [22, 23] and [24]).

Now we illustrate conclusions of Theorem 4.1 and Theorem 4.3 by a simple example involving a type of delay not considered yet (to avoid a discussion on zeros of $Q(\lambda)$, we put $k=1)$.

Example 4.6 Let $y$ be a solution of the dynamic equation

$$
y^{\nabla}(t)=a y(t)+b y\left(t^{1 / 2}\right), \quad t \in \mathbb{T},
$$

where $a, b$ are nonzero real scalars. Because of a type of $\tau$, we assume that $\mathbb{T}$ has a minimum $m=1$. Then $q=1 / \tau^{\prime}(m)=2$ and the corresponding Schröder equation (4.2) becomes

$$
\varphi\left(t^{1 / 2}\right)=\frac{1}{2} \varphi(t)
$$

which admits the solution $\varphi(t)=\log _{2} t$ having the required properties. We distinguish two cases: 
(i) If $\mathbb{T}=\mathbb{R}($ more precisely $\mathbb{T}=[1, \infty)$ ) and $a<0$, then by Theorem 4.1

$$
y(t)=O\left(\left(\frac{|b|}{-a}\right)^{\log _{2} \log _{2} t}\right) \quad \text { as } t \rightarrow \infty .
$$

(ii) If $\mathbb{T}=\left\{2^{2^{n}}: n \in \mathbb{Z}\right\} \cup\{1\}$, then (4.5) becomes (4.14) and by Theorem 4.3

$$
y(t)=c\left(-\frac{b}{a}\right)^{\log _{2} \log _{2} t}+O\left(\left(\left|\frac{b}{a}\right|-\varepsilon\right)^{\log _{2} \log _{2} t}\right) \quad \text { as } t \rightarrow \infty
$$

where $c \in \mathbb{R}$ and $0<\varepsilon<|b / a|$ are suitable scalars.

Remark 4.7 Equation (4.13) with $\mathbb{T}=\mathbb{R}$ has been studied in [25] as the differential equation with advanced power argument, i.e. when $\tau(t)=t^{\gamma}, \gamma>1$ (for extensions to the case of a general advanced argument $\tau$, see also [26]). It is interesting to observe that asymptotic formulae derived in these papers are very close to the property (4.15).

\section{Concluding remarks}

Comparing results of Theorem 4.1 and Theorem 4.3 (resp. Corollary 4.4), one can observe close similarities between stability and asymptotic properties of the differential equation (4.1) and its dynamic discrete analogue (2.8). As regards their proofs, some common ideas have been involved especially in the proof of Theorem 3.1, but Theorems 4.1 and 4.3 themselves had to be proved separately. In particular, the proof procedure of Theorem 4.3 is not applicable to the continuous case, at least in the presented form. Our conjecture is that the asymptotic estimate (4.12), which is slightly stronger that (4.3), is valid (perhaps in a modified form) also for the corresponding differential equation (4.1). In particular, we believe that the zero analysis of $Q(\lambda)$ instead of $\tilde{Q}(\lambda)$ should be involved here similarly as in the discrete case. However, an issue of the joint proof of such a property on a general time scale (including the continuous one) seems to be a difficult task.

\section{Competing interests}

The author declares that he has no competing interests.

\section{Acknowledgements}

The research was supported by the grant P201/11/0768 of the Czech Science Foundation and by the project FSI-S-11-3 of Brno University of Technology. The author is grateful to the referee for his (or her) suggestions and comments.

Received: 4 February 2013 Accepted: 9 May 2013 Published: 31 May 2013

\section{References}

1. Agarwal, RP, Anderson, DR, Zafer, A: Interval oscillation criteria for second-order forced delay dynamic equations with mixed nonlinearities. Comput. Math. Appl. 59(2), 977-993 (2010)

2. Anderson, DR: Global stability for nonlinear dynamic equations with variable coefficients. J. Math. Anal. Appl. 345(2), 796-804 (2008)

3. Anderson, DR, Kenz, Z: Global asymptotic behavior for delay dynamic equations. Nonlinear Anal. 66, 1633-1644 (2007)

4. Bohner, M: Some oscillation criteria for first order delay dynamic equations. Far East J. Appl. Math. 18(3), 289-304 (2005)

5. Čermák, J, Urbánek, M: On the asymptotics of solutions of delay dynamic equations on time scales. Math. Comput. Model. 46, 445-458 (2007)

6. Diblík, J, Rưžičková, I: Combination of Lyapunov and retract methods in the investigation of the asymptotic behavior of solutions of systems of discrete equations. Dyn. Syst. Appl. 18, 507-538 (2009)

7. Medved', M, Škripková, L: Sufficient conditions for the exponential stability of delay difference equations with linear parts defined by permutable matrices. Electron. J. Qual. Theory Differ. Equ. 2012, 22 (2012)

8. Bohner, M, Peterson, A: Dynamic Equations on Time Scales - An Introduction with Applications. Birkhäuser, Boston (2001) 
9. Bohner, M, Peterson, A: Advances in Dynamic Equations on Time Scales. Birkhäuser, Boston (2003)

10. Čermák, J, Kundrát, P, Urbánek, M: Delay equations on time scales: essentials and asymptotics of the solutions. J. Differ. Equ. Appl. 14(6), 567-580 (2008)

11. Elaydi, S: An Introduction to Difference Equations, 3rd edn. Undergraduate Texts in Mathematics. Springer, New York (2005)

12. Freedman, HI, Kuang, Y: Stability switches in linear scalar neutral delay equations. Funkc. Ekvacioj 34, 187-209 (1991)

13. Marden, M: Geometry of Polynomials. Mathematical Surveys and Monographs, vol. 3. Am. Math. Soc., Providence (1966)

14. Iserles, A: On the generalized pantograph functional-differential equation. Eur. J. Appl. Math. 4, 1-38 (1992)

15. Iserles, A: Exact and discretized stability of the pantograph equation. Appl. Numer. Math. 24, 295-308 (1997)

16. Kato, T, McLeod, JB: The functional-differential equation $y^{\prime}(x)=a y(\lambda x)+b y(x)$. Bull. Am. Math. Soc. 77, $891-937$ (1971)

17. Neuman, F: On transformations of differential equations and systems with deviating argument. Czechoslov. Math. J. 31(106), 87-90 (1981)

18. Neuman, F: Transformations and canonical forms of functional-differential equations. Proc. R. Soc. Edinb. A 115, 349-357 (1990)

19. Jaroš, J: An application of change of independent variable in the oscillation theory of differential equations with unbounded delays. Acta Math. Univ. Comen. LVIII-LIX, 99-106 (1990)

20. Kuczma, M, Choczewski, B, Ger, R: Iterative Functional Equations. Encyclopedia of Mathematics and Its Applications. Cambridge University Press, Cambridge (1990)

21. Agarwal, RP, Pituk, M: Asymptotic expansions for higher-order scalar difference equations. Adv. Differ. Equ. 2007 Article ID 67492 (2007)

22. Dannan, FM: The asymptotic stability of $x(n+k)+a x(n)+b x(n-l)=0$. J. Differ. Equ. Appl. 10(6), $589-599$ (2004)

23. Kuruklis, SA: The asymptotic stability of $x_{n+1}-a x_{n}+b x_{n-k}=0$. J. Math. Anal. Appl. 188, 719-731 (1994)

24. Čermák, J, Jánský, J, Kundrát, P: On necessary and sufficient conditions for the asymptotic stability of higher order linear difference equations. J. Differ. Equ. Appl. 18(11), 1781-1800 (2012)

25. Heard, ML: Asymptotic behavior of solutions of the functional differential equation $x^{\prime}(t)=a x(t)+b x\left(t^{\alpha}\right), \alpha>1$. J. Math. Anal. Appl. 44, 745-757 (1973)

26. Čermák, J: Asymptotic properties of differential equations with advanced argument. Czechoslov. Math. J. 50(4), $825-837(2000)$

doi:10.1186/1687-1847-2013-155

Cite this article as: Čermák: Some qualitative properties of linear dynamic equations with multiple delays. Advances in Difference Equations 2013 2013:155.

\section{Submit your manuscript to a SpringerOpen ${ }^{\circ}$ journal and benefit from:}

- Convenient online submission

Rigorous peer review

- Immediate publication on acceptance

- Open access: articles freely available online

- High visibility within the field

- Retaining the copyright to your article 